\title{
COMMUTATIVE REGULAR RINGS WITH INTEGRAL CLOSURE
}

\author{
BY
}

L. LIPSHITZ ${ }^{1}$

ABSTRACT. First order conditions are given which are necessary

for a commutative regular ring to have a prime integrally closed extension. If the ring is countable these conditions are also sufficient.

In [8] an example was given of a commutative regular ring with no prime model extension to a commutative integrally closed regular ring. In this paper we give (in \$2) first order conditions which are necessary for a commutative regular ring, $R$, to have a prime extension to an integrally closed regular ring. In the case that $R$ is countable these conditions are also sufficient (\$3). They are, however, not sufficient in the case that $R$ is uncountable (\$4).

In [1] and [5] (see also [6] and [9]) it was shown that the theory $K_{\overline{C R}}^{*}$ of integrally closed commutative regular rings without minimal $(\neq 0)$ idempotents is the model completion of the theory of commutative regular rings, $K_{C R}$. In $\$ 5$ we show that a model of $K_{C R}$ has a prime extension to a model of $K_{\overline{C R}}$ (the theory of integrally closed commutative regular rings) if and only if it has a prime extension to a model of $K^{*} \frac{}{C R}$.

1. Preliminaries. A commutative ring $R$ with unit is called regular (in the sense of von Neumann) if $R$ satisfies $\forall x y^{y}\left(x^{2} y=x\right) . B_{R}$ is the Boolean algebra of idempotents of $R$. The operations of $B_{R}$ are $e_{1} \cup e_{2}=e_{1}+e_{2}-$ $e_{1} e_{2}$ and $e_{1} \cap e_{2}=e_{1} e_{2} . S_{R}$ is the maximal ideal space of $R$. It is well known that if $R$ is regular and commutative then $S_{R}$ is the Stone space of $B_{R}$. In a natural way $R$ is a ring of functions on $S_{R} \cdot(R \ni a \rightarrow a(\cdot)$ defined by $a(s)=a+s$ in $R / s$, for $s \in S_{R}$ any maximal ideal of $R$. If $R$ is regular then $R / s$ is a field.) It will be intuitively helpful to think of commutative regular rings as rings of functions in this way. If $R$ is a commutative regular

Received by the editors July $14,1974$. $02 \mathrm{H} 15$.

AMS (MOS) subject classifications (1970). Primary 13L05, 13B20; Secondary

Key words and phrases. Commutative regular rings, integrally closed rings, prime model extensions.

1 This research supported in part by N.S.F. grant GP 43749. 
ring and $e(\neq 0)$ is an idempotent of $R$ then $e$ is a $\{0,1\}$ function on $S_{R}$. There is a one-to-one correspondence between clopen subsets of $S_{R}$, and idempotents of $R$ given by $e \in R$ correspond to $\left\{s \in S_{R} \mid e(s)=1\right\}=E_{e}$. We say that a relation among elements of $R$ holds on idempotent $e$ if for every $s \in E_{e}$ the relation is true mod $s$. We say that a relation holds at a point $s \in S_{R}$ if it is true mod $s$ in $R / s$.

$K_{C R}$ is the theory of commutative regular rings. $K_{\overline{C R}}=K_{C R} \cup$ \{every monic polynomial has a root $\}$ is the theory of integrally closed regular rings. $K_{C R}^{*}=K_{C R} \cup\{$ there are no minimal $(\neq 0)$ idempotents $\}$ and $K_{\overline{C R}}^{*}=K_{\overline{C R}} \cup$ \{there are no minimal $(\neq 0)$ idempotents\} (cf. [5]).

If $T_{1}$ and $T_{2}, T_{1} \subset T_{2}$, are two theories and $\mathscr{Q} \vDash T_{1}, \mathfrak{Q} \subset \mathfrak{R} \vDash T_{2}$ then we call $\mathfrak{R}$ a prime model extension of $\mathscr{U}$ to a model of $T_{2}$ if whenever $\mathfrak{Z}^{\prime}$ $\vDash T_{2}$ and $\phi: \mathscr{U} \rightarrow \Omega^{\prime}$ is an embedding, there is an extension of $\phi$ to an

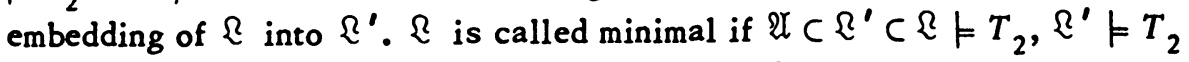
implies $\mathfrak{I}^{\prime}=\mathfrak{\Omega}$. It is well known (cf. [7]) that if $\mathfrak{Z}$ is a minimal prime model extension of $\mathscr{U}$ to a model of $T_{2}$, then every prime model extension of $\mathscr{U}$ to a model of $T_{2}$ is isomorphic with $?$.

If $R \vDash K_{C R}$ and $\bar{R}$ is a prime model extension of $R$ to a model of $K_{\overline{C R}}$ then we call $\bar{R}$ an integral closure of $R$. It will be shown (Proposition 1) that such an $\bar{R}$ is in fact minimal and hence is the unique integral closure of $R$.

If $p(x) \in R[x], R \vDash K_{C R}$, then $p$ is called irreducible if $p(x)$ is irreducible at every point $s \in S_{R}$, i.e. if for every $s \in S_{R}$ there are no polynomials $p_{1}$ and $p_{2} \in R[x]$ such that $p(x)=p_{1}(x) p_{2}(x)$ at $s$ and degree $\left(p_{1}(x)\right)$ and degree $\left(p_{2}(x)\right)>0$ at $s . p(x)$ is called unambiguous if, at each $s \in S_{R}$, $p(x)$ is a power of a polynomial irreducible at $s$, of degree $>0$ at $s$. It is shown in [s] that if $\phi\left(a_{1}, \ldots, a_{n}\right)$ is any quantifier free relation among $a_{1}$, $\ldots, a_{n} \in R \vDash K_{C R}$ then there is an idempotent $e \in R$ such that $\phi$ holds at every point of $E_{e}$ and fails at every point of $S_{R}-E_{e}=E_{1-e} \cdot$ Using this is not hard to see that the Euclidean algorithm holds in $R$ and hence that if $p(x), q(x) \in R[x]$ then $(p, q)(x)=(p(x), q(x))$, the g.c.d. of $p(x)$ and $q(x)$, is an element of $R[x]$. Using in addition the compactness of $S_{R}$ it follows also that if $p(x) \mid q(x)$ at each point of $S_{R}$ then $p(x) \mid q(x)$ in $R[x]$. In obtaining $(p, q)$ from $p$ and $q$ it may be necessary to consider different cases of various coefficients vanishing or not on (a finite number of) different idempotents. This will often be the case in the arguments which follow. We shall not usually make explicit mention of this.

If $k \in \mathbf{N}$ let $e_{k}$ be the idempotent of $R$ on which $k(=1+\cdots+1)=$ 0 holds (and $k \neq 0$ holds on $1-e_{k}$ ). Let $p(x) \in R[x]$ be unambiguous and 
monic of degree $n>0$. On $1-e_{n !}$, by considering $\left(p(x), p^{\prime}(x)\right)=p_{1},\left(p_{1}(x), p_{1}^{\prime}(x)\right)$ $=p_{2}(x)$ etc. one can find an irreducible polynomial $q_{1}(x)$ such that $q_{1}(x) \mid p(x)$ on $1-e_{n !}$. This procedure may fail on $e_{n !}$, since at some point of $e_{n !}$ we may have $p^{\prime}(x)=0$ (or $p_{1}^{\prime}(x)=0$ etc.). We can, however, find a polynomial $q_{2}(x)$ on $e_{n !}$ such that if $s \in E_{e_{n !}}$ and $\operatorname{char}(R / s)=k$ then, at $s, p(x)=q_{2}\left(x^{k^{m}}\right)$, for some $m \in \mathrm{N}$, and $q_{2}(x)$ is unambiguous on $e_{n !}$. (Let $e_{k}^{\prime}$ be the subidempotent of $e_{k}$ on which $p^{\prime}(x)=0$. On $e_{k}^{\prime}$ replace $x^{k}$ by $x$ in $p(x)$ to get $p_{1}(x)$. After a finite number of such steps one will obtain the required polynomial $q_{2}(x)$.)

Let $T=K_{C R} \cup$ \{every monic polynomial of degree $n$ has an unambiguous factor $\mid n \in \mathrm{N}\}$.

For terms used but not defined in this paper the reader is referred to [5] or [7]. Good references for the algebra in this paper are [2] and [3].

2. Necessity. In this section we show that if $R \models K_{C R}$ then for $R$ to have a prime extension, $\bar{R}$, to a model of $K_{\overline{C R}}$ it is necessary that $R \vDash T$. We also show that if such an $\bar{R}$ exists then it is unique, up to isomorphism.

Lemma 1. If $R, R_{1} \vDash K_{C R}, R \subset R_{1}$ and $\alpha \in R_{1}$ satisfies $p(\alpha)=0$ for some $p(x) \in R[x]$ then every maximal ideal of $R[\alpha]$ is of the form $(s \cup\{q(\alpha)\})$, $s \in S_{R}$ with $q(x) \mid p(x)$ at $s$ and $q(x) \in R[x]$ irreducible at $s$.

Proof. Let $s^{\prime} \in S_{R}[a]$. Then $s^{\prime} \cap R=s \in S_{R}$. At $s$ let $p(x)=p_{1}^{k}(x)$ $\ldots p_{m}^{k}(x)$ with $\left(p_{i}, p_{j}\right)=1$ at $s$. Then $p_{1}(a) \ldots p_{m}{ }^{k}(a)=0$ so, since $s^{\prime}$ is maximal and hence prime, $q(\alpha)=p_{i}(\alpha) \in s^{\prime}$ for some $i$. We must show that $s \cup\{q(a)\}$ generates $s^{\prime}$. Let $a \in s^{\prime}$. Then either $a e=a$ for some idempotent $e \in s$ or $a(1-e) \in R[\alpha]-R$ for every idempotent $e \in s$. In the first case $a \in s$. In the second $a(1-e)=r(a)(1-e)$ with degree $(r(x))>0$ at $s$. Then $r(a), q(a) \in s^{\prime}$ so $(r, q)(\alpha) \in s^{\prime}$, so $(r, q) \neq 1$ at $s$, i.e., $q(x) \mid r(x)$ at $s$ and hence on some idempotent $1-e, e \in s$. Hence $a \in(s \cup\{q(a)\})$.

Lemma 2. If $R, a$ are as above then $R[\alpha] \models K_{C R}$.

Proof. Let $S^{\prime}$ be the maximal ideal space of $R[\alpha]$ and let $q(\alpha) \in R[\alpha]$. Without loss of generality we may assume that $\operatorname{degree}(q)<\operatorname{degree}(p)=n$.

Let $u(x)=\left(p(x), q^{n}(x)\right)$ and let $v(x)=p(x) / u(x)$. Then $(v(x), q(x))=1$ and $p(x) \mid v(x) q^{n}(x)$ so if $y \in S^{\prime}, q(\alpha) \notin y$ if and only if $v(\alpha) \in y$. Since $(q(x), v(x))=1$ there exist polynomials $w_{1}(x), w_{2}(x)$ such that $q(x) w_{1}(x)+$ $v(x) w_{2}(x)=1$. Then $q^{2}(\alpha) w_{1}(\alpha)=q(\alpha)-q(\alpha) v(\alpha) w_{2}(\alpha)$. But $q(\alpha) v(\alpha)=0$ in $R[\alpha]$ so $q^{2}(\alpha) w_{1}(\alpha)=q(\alpha)$, i.e. $R[\alpha] \vDash K_{C R}$.

Let $\phi: R_{1} \rightarrow R_{2}$ be an embedding and let $R_{i} \vDash K_{C R}$ and $S_{i}$ be the 
Stone space of $R_{i}(i=1,2)$. Define $\phi^{*}: S_{2} \rightarrow S_{1}$ by $\phi^{*}\left(s^{\prime}\right)=\phi^{-1}\left(s^{\prime}\right)$, $s^{\prime} \in S_{2}$. Then $\phi^{*}$ is a continuous mapping of $S_{2}$ onto $S_{1}$. If $s \in S_{1}$ we say $s$ splits in $R_{2}$ if $\phi^{*-1}(s)$ contains more than one point of $S_{2}$.

Lemma 3. (i) If $R, a, p$ are as above with $p$ irreducible at $s$ then $s$ does not split in $R[a]$ and if $N$ is a neighborbood of $s$ (id*-1 $(s)$ ) in $S_{R}[a]$ then there is a neighborbood $M$ of $s$ in $S_{R}$ such that $\mathrm{id}^{*-1}(M) \subset N$. (id: $R \rightarrow R[a]$.)

(ii) If $\bar{R}$ is a prime extension of $R$ to a model of $K_{\overline{C R}}$ then no $s \in S_{R}$ splits in $\bar{R}$.

Proof. (i) The fact that $s$ does not split in $R[a]$ follows from the irreducibility of $p(x)$ at $s$ by Lemma 1 . Let $N$ be clopen in $S_{R[a]}$. Then $N$ is defined by some idempotent $q(a) \in R[a]$ by $N=\left\{s^{\prime} \in S_{R}[a] \mid q(a)\left(s^{\prime}\right)=0\right\}$. Hence $p(x) \mid q(x)$ at $s$ and hence in some neighborhood $M$ of $s$. Clearly $\mathrm{id}^{*-1}(M) \subset N$.

(ii) Let $s \in S_{R}$. There is an $R_{1} \vDash K_{\overline{C R}}$ with $R \subset R_{1}$ such that $s$ does not split in $R_{1}$. $R_{1}$ can be obtained by repeatedly adjoining roots of polynomials irreducible at $s$ (more precisely $\left(\mathrm{id}^{*}\right)^{-1}(s)$ ). Since $\bar{R}$ is prime over $R$ we have $R \stackrel{\text { id }}{\longrightarrow} \bar{R} \stackrel{\phi}{\rightarrow} R_{1}$. Since $s$ does not split in $R_{1},(\phi \circ \mathrm{id})^{*-1}(s)$ contains only one point of $S_{R_{1}}$. So id ${ }^{*-1}(s)$ contains only one point of $S_{\bar{R}}$.

Remark. If $R \subset \bar{R}$ are models of $K_{C R}$ and no point of $S_{R}$ splits in $\bar{R}$ then $\mathrm{id}^{*}$ is a homeomorphism and hence $B_{R}=B_{\bar{R}}$, i.e. $R$ and $\bar{R}$ have the same idempotents.

Proposition 1. If $R \vDash K_{C R}$ and $\bar{R}$ is a prime extension of $R$ to a model of $K_{\overline{C R}}$ then $\bar{R}$ is minimal and bence unique.

Proof. From the above remark $R$ and $\bar{R}$ have the same idempotents. Every element of $\bar{R}$ is algebraic over $R$, i.e. satisfies some $p(x)=0$ for $p(x) \in R[x]$. Suppose $R \subset R_{1} \subset \bar{R}$ with $R_{1} \vDash K_{\overline{C R}}$. Let $\alpha \in \bar{R}$ satisfy $p(\alpha)=$ $0, p(x) \in R[x]$. Let $p(x)=\Pi\left(x-\beta_{i}\right)$ in $R_{1}$. Then at each point of $S_{\bar{R}}=S_{R_{1}}$ $=S_{R}$, a equals one of the $\beta_{i}$. Then there are idempotents $e_{i} \in B_{\bar{R}}=B_{R}$ such that $a=\Sigma e_{i} \beta_{i}$. Hence $\alpha \in R_{1}$, i.e. $R_{1}=\bar{R}$.

Lemma 4. If $R \vDash K_{C R}, R \notin T$ then there is a polynomial $p(x) \in R(x)$ and a point $s \in S_{R}$ such that $p(x)$ is irreducible at $s$ but in every neighborbood $N$ of $s p(x)$ is ambiguous.

Proof. Let $p(x)$ be a monic polynomial of lowest degree not having an unambiguous factor. Let $X=\left\{s \in S_{R} \mid\right.$ at $s, p(x)$ is ambiguous $\}$. It is clear that $X$ is open since if $p(x)=p_{1}(x) p_{2}(x)$ with $\left(p_{1}(x), p_{2}(x)\right)=1$ at $s$ then 
this holds in some neighborhood of $s . X$ cannot be closed. For if it were, $X$ would be compact and equal to $E_{e}$ for some idempotent $e$. Using the compactness of $X$ we could factor $p(x)=q(x) r(x)$ on $X(=E e)$ with both $q(x)$ and $r(x)$ having positive degree everywhere on $X$. Then at least one of $q(x)$, $r(x)$, say $q(x)$, would not have an unambiguous factor on $X$. There will be a subidempotent $e_{1}$ of $e$ on which $q(x)$ is monic and does not have an unambiguous factor. Let $q(x)$ have degree $m$ on $e_{1}$. Then $p_{1}(x)=q(x) e_{1}+$ $x^{m}\left(1-e_{1}\right)$ does not have an unambiguous factor, is monic and is of lower degree than $p(x)$. This would contradict the way $p(x)$ was chosen. Consequently $\bar{X}-X \neq \varnothing$. Let $s \in \bar{X}-X$.

Theorem 1. If $R \vDash K_{C R}$ and $R \forall T$ then $R$ does not have a prime extension to a model of $K_{\overline{C R}}$.

Proof. Let $R_{1}$ be a candidate for such an extension. Then by the remark following Lemma $3, B_{R_{1}}=B_{R}$ and $S_{R_{1}}=S_{R}$. Let $s, p(x)$ be as in Lemma 4. Since we are only interested in $p(x)$ in a neighborhood of $s$ we can take $p(x)$ to be monic. Let $p(x)=\Pi_{i=1}^{n}\left(x-a_{i}\right)$ in $R_{1}$. Let $X=\left\{x \in S_{R} \mid p\right.$ is ambiguous at $s\}$. Then as in the proof of Lemma $4, X$ is open. Let $X_{1}$, $\ldots, X_{n-1}$ be disjoint copies of $X$, say $X_{i}=\phi_{i}(X)$, the $\phi_{i}$ homeomorphisms, and let $S^{\prime}=S \cup X_{1} \cup \cdots \cup X_{n-1}$. We define a topology on $S^{\prime}$ by specifying the basic neighborhood of points of $S^{\prime}$. If $s \in X$, a basic neighborhood for $s$ is any open subset of $X$ containing $s$. Similarly if $s \in X_{i}$. If $s \in S-\bar{X}$, a basic neighborhood of $s$ is any open subset of $S-\bar{X}$ containing $s$. If $s \epsilon$ $\bar{X}-X$ the basic neighborhoods of $s$ are of the form $N \cup \cup_{i=1}^{n-1} \phi_{i}(N \cap X)$ where $N$ is a neighborhood of $s$ in $S$.

It is clear that $S^{\prime}$ is a totally disconnected compact Hausdorff space containing $S$. All we have done is glued on $n-1$ copies of $X$ along the boundary of $X . R\left[a_{1}, \ldots, a_{n}\right]$ can be considered as a ring of functions on $S^{\prime}$ as follows: If $a \in R\left[\alpha_{1}, \ldots, \alpha_{n}\right]$ then $a(s)$ is defined for $s \in S$, if $s \epsilon$ $X_{i}$ let $a(s)=a\left(\phi_{i}^{-1}(s)\right)$. Let $a^{\prime}$ be the following function on $s^{\prime}$.

$$
\alpha^{\prime}= \begin{cases}\alpha_{1} & \text { on } S, \\ \alpha_{i+1} & \text { on } X_{i}\end{cases}
$$

Consider $R\left[a^{\prime}\right]$. Since $p$ is irreducible at $s$ and $p\left(\alpha^{\prime}\right)=0, s$ does not split in $R\left[\alpha^{\prime}\right]$. Also by Lemma $2, R\left[\alpha^{\prime}\right] \vDash K_{C R}$. By repeatedly adjoining roots of polynomials which are irreducible at $s$ we can find $R_{2} \vDash K_{\overline{C R}}$ with $R_{2} \supset$ $R\left[\alpha^{\prime}\right]$ and $s$ not splitting in $R_{2}$. We shall show that there is no enbedding $\phi: R_{1} \rightarrow R_{2}$. Suppose there were. $\phi\left(\alpha_{1}\right), \ldots, \phi\left(\alpha_{n}\right)$ are roots of $p$ in $R_{2}$ 
with $p(x)=\Pi_{i=1}^{n}\left(x-\phi\left(\alpha_{i}\right)\right)$ in $R_{2} \cdot \alpha^{\prime}$ is a root of $p$ in $R_{2}$ so there are idempotents $e_{i}$ in $R_{2}$ such that, on $e_{i}, \alpha^{\prime}=\phi\left(\alpha_{i}\right)$ and $\bigcup E_{e_{i}}=S_{R_{2}}$. Hence in some neighborhood, $N$ of $s$ in $S_{R_{2}}, \alpha^{\prime}=\phi\left(\alpha_{1}\right)$, say. By Lemma 3 there is a neighborhood $M$ of $s$ in $S_{R}$ such that $\phi^{*-1}(M) \subset N$. Let $s_{1}$ be a point of $M$ at which $p$ is ambiguous, say $p(x)=p_{1}(x) p_{2}(x),\left(p_{1}(x), p_{2}(x)\right)=1$ at $s_{1}$ and suppose $p_{1}\left(a_{1}\right)=0$ at $s_{1} \in S_{R_{1}}=S_{R_{1}}$. Hence $p_{1}\left(\phi\left(a_{1}\right)\right)=0$ at each point of $\phi^{*-1}\left(s_{1}\right)$. But at some point of $\phi^{*-1}\left(s_{1}\right), p_{2}\left(a^{\prime}\right)=0$ and hence $p_{1}\left(\alpha^{\prime}\right) \neq 0$. This contradiction shows that no such $\phi$ exists.

3. Sufficiency in the countable case. In this section we show that if $R \models T, \overline{\bar{R}}=\boldsymbol{\aleph}_{0}$ then $R$ has a prime extension to a model of $K_{\overline{C R}}$.

Lemma 5. If $R \neq K_{C R}$ and $p(x) \in R[x]$ is unambiguous and $R\left[a_{i}\right] \subset R_{i}$ $\vDash K_{C R}$ with $p\left(\alpha_{i}\right)=0$ in $R_{i}$ for $i=1,2$ then $R\left[\alpha_{1}\right] \simeq R\left[\alpha_{2}\right]$.

Proof. Since $p(x)$ is unambiguous $S_{R}=S_{R\left[a_{1}\right]}=S_{R\left[a_{2}\right]}$ and hence $R\left[\alpha_{1}\right], R\left[\alpha_{2}\right]$ are both rings of functions on $S_{R}$. The canonical mapping taking $\alpha_{1} \rightarrow \alpha_{2}$ gives an isomorphism at each point, $s \in S_{R}$ and hence gives an isomorphism of $R\left[\alpha_{1}\right]$ and $R\left[\alpha_{2}\right]$.

Lemma 6. If $R \vDash T$ and $p(x) \in R[x]$ is unambiguous and $p(\alpha)=0$ then $R[\alpha] \vDash T .\left(R[\alpha] \subset R^{\prime} \vDash K_{\overline{C R}}.\right)$

Proof. We must prove that if $q(x)$ is a polynomial in $R[a][x]$ then $q$ has an unambiguous factor over $R[\alpha]$. Let $q=q(\alpha, x)$ and let $q_{1}(x)=$ $\Pi_{i=1}^{n} q\left(\alpha_{i}, x\right)$. (The $\alpha_{i}$ are distinct roots of $p$ in some extension, $R^{\prime}$ of $R[\alpha]$ in which $p$ splits. Since $q_{1}$ is symmetric in the $a_{i}, q_{1}$ is actually a polynomial in $R[x]$, since every symmetric polynomial of the $\alpha_{i}$ 's is a polynomial of the coefficients of $p(x)$.) Let $q_{2}(x)$ be an unambiguous factor of $q_{1}(x)$ in $R[x]$ such that $\left(q_{2}, q\right) \neq 1$ at any point $s \in S$. We shall show that in $R[\alpha]$ $q_{2}$ can be factored as a product of unambiguous polynomials. This will give the desired result for $q_{1}$.

First we remark that we can take $p$ and $q_{2}$ to be irreducible and separable. For let $p_{s}$ and $q_{s}$ be the irreducible separable polynomials corresponding to $q$ and $q_{2}$ as in $\S 1$, and suppose that $p_{s}(\beta)=0$ and that $q_{s}$ is a product of unambiguous factors in $R[\beta]$. It is clear that $q_{2}$ is then a product of unambiguous factors in $R[\beta]$ and that these factors remain unambiguous in in $R[a]$. (This follows at each point of $S_{R}$ from elementary theorems about fields.)

Hence we must show that if $p(x), q(x) \in R[x]$ are irreducible and $p(\alpha)=$ 0 then in $R[a], q(x)$ is a product of unambiguous (in fact irreducible) factors. 
Let $p(x)$ be monic of degree $n$ and $q(x)$ monic of degree $m$. This may involve considering different cases on different idempotents. This is no problem since if $e \in R$ is an idempotent then $R=e R \oplus(1-e) R$. Let

$$
T(\mathrm{t}, \mathbf{u}, x)=\prod_{\sigma \in S_{n} ; \nu \in S_{m}}\left(x-\sum t_{i} a_{\sigma(i)}-\sum u_{j} \beta_{\nu(j)}\right)
$$

where the $\alpha_{i}$ 's are distinct roots of $p$ and the $\beta_{j}$ 's distinct roots of $q$ and $S_{n}$ is the symmetric group on $n$ letters, $S_{m}$ the symmetric group on $m$ letters and the $t_{i}, u_{j}$ are new variables. $T(t, u, x)$ is a polynomial over $R$ since it is symmetric in the $\alpha_{i}$ and $\beta_{j}$. Let $\mu=m ! n !+1$, and let $T(x)=\Pi T(t, u, x)$ where the product is taken over all vectors $t$ and $u$ with entries from the set $\{1,2, \ldots, \mu\}$, if they are distinct. Recall that $e_{p}$ is the idempotent on which $p=0$ holds. On $e_{p}$ for $p<\mu$ these elements will not be distinct, so we must consider what happens on these idempotents separately. Let $e_{p, k}$ be the subidempotent of $e_{p}$ on which every coefficient of $p(x)$ and $q(x)$ belongs to the finite field with $p^{k}$ elements (i.e. satisfies $t^{p^{k}}-t=0$ ). On each $e_{p, k}$ the lemma follows by considering a finite number of different cases, each of which behaves just like a finite field. On $f=e_{p}-\bigcup_{p} k_{\leq \mu} e_{p, k}$ there is a coefficient $a$ (of $p(x)$ or $q(x)$ ) such that $a, a^{2}, \ldots, a^{\mu}$ are distinct at every point of $f$. On this idempotent we can use this set of distinct elements instead of $\{1,2, \ldots, \mu\}$.

Let $T(x)=r(x) \Pi_{i}(x)$ be an unambiguous (in fact irreducible) factoring of $T$ with degree $(r) \geq \operatorname{degree}\left(r_{i}\right)$ for all $i$. Let $\delta$ be a root of $r$. Since $r$ is unambiguous $S_{R[\delta]}=S_{R}$. At each point $s \in S_{R}, p(x)$ and $q(x)$ split in $R[\delta]$. This is clear since the degree $[R[\delta] / s: R / s]$ will be the degree of the splitting field $F$ of $p(x)$ and $q(x)$ at $s$ over $R / s$, and $R[\delta] / s \subset F$. Notice that if $w(\delta)$ is a root of $p$ (or $q$ ) at $s$ then $w(\delta)$ is a root of $p$ (or $q$ ) in some neighborhood of $s$. Since $S_{R}$ is compact we can find $a_{i}(\delta), \beta_{i}(\delta) \epsilon$ $R[\delta]$ such that in $R[\delta] p(x)=\prod_{i=1}^{n}\left(x-\alpha_{i}(\delta)\right)$ and $q(x)=\prod_{i=1}^{n}\left(x-\beta_{i}(\delta)\right)$. The $\alpha_{i}$ and $\beta_{i}$ are polynomials in $\delta$. Consider the following sets

$$
V_{i j}=\left\{1, \alpha_{1}^{2}, \ldots, \alpha_{1}^{n-1}, \beta_{i}, \beta_{i}^{2}, \ldots, \beta_{i}^{j}\right\} .
$$

Each element of $V_{i j}$ is a linear (over $R$ ) combination of $1, \delta, \delta^{2}, \ldots, \delta^{k-1}$ ( $k=$ degree $r$ ) which are linearly independent over $R$. Hence for each $i, j$ there is an idempotent $e_{i j}$ on which the set $V_{i j}$ is linearly dependent over $R$. $e_{i j}$ can be found as the set where suitable determinants vanish. On $e_{i, j+1}-e_{i j}, \beta_{i}$ satisfies an irreducible polynomial of degree $j+1$ over 
$R\left[a_{1}\right]$. Also $e_{i, m-1}=1$. It is now easy to see that we can piece together irreducible factors of $q(x)$ over $R\left[\alpha_{1}\right]$. This proves the lemma.

Theorem 2. If $R \models T$ and $\overline{\bar{R}}=\gamma_{0}$ then $R$ has a prime extension to $a$ model of $K_{\overline{C R}}$.

Proof. Let $\bar{R}=R\left[a_{i} \mid i<\omega\right]$ be such that $a_{i+1}$ satisfies an unambiguous polynomial over $R\left[a_{1}, \ldots, \alpha_{i}\right]$ and $\bar{R} \vDash K_{\overline{C R}}$. Since $R$ is countable it is clear that such an $\bar{R}$ exists by Lemma 6 . Lemma 5 shows that $\bar{R}$ is prime over $R$, since $R\left[\alpha_{1}, \ldots, a_{i}\right]\left[\alpha_{i+1}\right]$ is prime over $R\left[a_{1}, \ldots, \alpha_{i}\right]$.

Remark. We have actually proved a little more than Theorem 2. We actually have that if $R \vDash T$ and $\left\{p_{i} \mid i \epsilon \omega\right\}$ is a countable set of polynomials in $R[x]$ then there is a prime way to extend $R$ to a model of $K_{C R}$ in which all the polynomials $p_{i}(x)$ split.

Theorems 1 and 2 together give the following result of [4].

Corollary 1. If $R \vDash K_{C R}$ and $\overline{\bar{R}}=\aleph_{0}$ then $R$ has a prime extension to a model of $K_{\overline{C R}}$ if and only if $R \vDash T$.

In this countable case the necessity of $T$ can be proved by using the elimination of quantifiers for $K_{\overline{C R}}^{*}$ (in the language with an additional function symbol $f$ defined by $\forall x\left(x^{2} f(x)=x \wedge x f^{2}(x)=f(x)\right)$, cf. [5]), and Ehrenfeucht's theorem.

4. The uncountable case. In this section we give an example of an $R F$ $T, \overline{\bar{R}}=2^{\boldsymbol{K}_{0}}$ without a prime extension to a model of $K_{\overline{C R}}$. Let $\overline{\mathbf{Q}}$ denote the algebraic closure of the rationals and let $\left\{x_{i} \mid i<\omega\right\}$ be algebraically independent. Let $F=\bar{Q}\left(x_{1}, x_{2}, \ldots\right)$ and let $\bar{F}$ be the algebraic closure of $F$. Let $R_{1}=F^{\omega}$ and $R_{2}$ be the subring of $R_{1}$ of all sequences which are constant except on finite sets. There exist $2^{\mathcal{X}_{0}}$ sequences $f_{\alpha}: \omega \rightarrow\left\{x_{i} \mid i<\omega\right\}$, $\alpha<2^{\boldsymbol{x}_{0}}$ such that if $\alpha \neq \beta\left(\alpha, \beta<2^{\boldsymbol{X}_{0}}\right)$ then range $\left(f_{\alpha}\right) \cap \operatorname{range}\left(f_{\beta}\right)$ is finite. Let $R_{3}=R_{2}\left[f_{\alpha} \mid \alpha<2^{N_{0}}\right]$ and let $R$ be the smallest regular subring of $R_{1}$ containing $R_{3}$. It is not hard to show that $B_{R}=B_{R_{2}}$ (= all finite or cofinite subsets of $\omega$ ) and that $R \neq T$. Let $R_{4}$ be the subring of $\bar{F}^{\omega}$ of all sequences which are constant except on finite sets. $R_{4}$ is the prime extension of $R_{2}$ to a model of $K_{\overline{C R}}$. Let $R_{s} \subset \bar{F}^{\omega}$ be a model of $K_{\overline{C R}}$ containing $R$, with $S_{R}=S_{R_{5}}(=\omega+1)$. We shall construct a model $R_{6} \supset R$ of $K_{\overline{C R}}$ such that there is no embedding of $R_{5} \rightarrow R_{6}$, over $R$. This will show that $R$ has no prime extension to a model of $K_{\overline{C R}}$.

Let $\left\{\phi_{a} \mid \alpha<2^{\aleph_{0}}\right\}$ be all the distinct embeddings of $R_{4}$ into $R_{4}$ over $R$. 
Notice that if $R_{4} \subset R_{6}$ and $\phi: R_{4} \rightarrow R_{6}$ is an embedding and $S_{R_{6}}=S_{R}$ then $\phi$ has at most one extension to an embedding $R_{5} \rightarrow R_{6}$ since if $a \in R_{5}$ then $a e_{i} \in R_{4}$ for each $e_{i}=\left(\delta_{i j}\right)_{j \in \omega}$ and $\phi(a)$ is determined by the $\phi\left(a e_{i}\right)$. For each $\alpha<2^{\boldsymbol{N}_{0}}$ choose $q_{a} \in \bar{F}^{\omega}$ such that $q_{a}^{2}=f_{a}$ and $\phi_{a}^{-1}\left(q_{a}\right) \notin R_{s}$ (i.e. there is no $b \in R_{\text {s }}$ such that $\left.\phi_{a}\left(b e_{i}\right)=q_{a} e_{i} \forall i \epsilon \omega\right)$. Such a $q_{a}$ exists since $R_{s}$ contains only countably many square roots of $f_{a}$. Let $R_{\sigma}$ be an extension of $R_{2}\left[q_{\alpha} \mid \alpha<2^{X_{0}}\right]$ to a model of $K_{\overline{C R}}$ with $S_{R_{\sigma}}=$ $S_{R}$. Suppose that $\phi: R_{5} \rightarrow R_{6}$ were an embedding. Then $\left.\phi\right|_{R_{4}}=\phi_{a}$ for some a. Let $x^{2}-f_{a}=\left(x-b_{1}\right)\left(x-b_{2}\right)$ in $R_{5}$. Let $E_{1}=\left\{s \in S_{R_{G}} \mid \dot{\phi}\left(b_{1}\right)(s)=q_{a}(s)\right\}$ and $E_{2}=S_{R}-E_{1}$. These must be clopen subsets of $S_{R_{6}}=S_{R_{5}}$ corresponding to idempotents $e_{1}$ and $e_{2}$, say. But then $q_{a}=\phi\left(b_{1}\right) e_{1}+\phi\left(h_{2}\right) e_{2}=$ $\phi\left(b_{1} e_{1}+b_{2} e_{2}\right)$ which is a contradiction.

The reason that $R$ has no prime extension to a model of $K_{\overline{C R}}$ is that there is too much interweaving among elements of $R$. It may be possible, by excluding this kind of interweaving, to arrive at some quite general sufficient condition for $R \vDash T$ to have a prime extension to a model of $K_{\overline{C R}}$.

5. Prime extensions to models of $K_{C R}^{*}$ and $K_{\overline{C R}}^{*}$. In this section we show that every model of $K_{C R}$ has a prime extension to a model of $K_{C R}^{*}$ and that if $R \models K_{C R}$ then $R$ has a prime extension to a model of $K_{\overline{C R}}$ if and only if $R$ has a prime extension to a model of $K_{\overline{C R}}^{*}$. of $K_{C R}^{*}$.

Proposition 2. Let $R \vDash K_{C R}$ then $R$ has a prime extension to a model

Proof. Let $X$ be the set of isolated points of $S_{R}$. For each $x \in X$ let $C_{x}$ be a copy of the Cantor set. Let $S_{R}^{*}$ be obtained from $S_{R}$ by replacing each $x \in X$ by $C_{x}$ (in the obvious way). $R$ can be considered as a ring of functions on $S_{R}^{*}$ (constant on each $C_{x}$ ). Let $B_{x}$ be the set of idempotents corresponding to clopen subsets of $C_{x}$. Let $B=\bigcup_{x \in X^{B}}$ and let $R^{*}=R[B]$. It is clear that $R \subset R^{*} \vDash K_{C R}^{*}$. We must show that $R^{*}$ is prime over $R$. So let $\phi: R \rightarrow R_{1} \vDash K_{C R}^{*}$. Let $e_{x}$ be the idempotent corresponding to $x \in X$. Observe that $B_{x}$ is the unique countable atom free Boolean algebra. Hence for each $x \in X^{x}$ there is a copy of $B_{x}$, say $B_{x}^{\prime}$ with $\phi\left(e_{x}\right)$ as largest element (i.e. $B_{x}^{\prime}$ is a Boolean algebra of subidempotents of $\phi\left(e_{x}\right)$ with $\phi\left(e_{x}\right)$ as 1 ). Let $B^{\prime}=\bigcup_{x \in X^{\prime}} B_{x}^{\prime}$. Then $\phi$ extends naturally to an embedding of $R^{*}$ onto $\phi(R)\left[B^{\prime}\right] \subset R_{1}$.

Proposition 3. If $R \vDash K_{C R}$ then $R$ has a prime extension to a model of 
$K_{\overline{C R}}^{*}$ if and only if $R$ has a prime extension to a model of $K_{\overline{C R}}$.

Proof. Proposition 2 shows that if $R$ has a prime extension to a model of $K_{\overline{C R}}$ then $R$ has a prime extension to a model of $K_{\overline{C R}}^{*}$. Conversely suppose that $R_{1}$ is a prime extension to a model of $K_{C R}^{*}$. Let $X \subset S_{R}$ be the set of isolated points of $S_{R}$ and let $e_{x}$ be the idempotent corresponding to $x \in X$. Let $\nu: R \rightarrow R_{1}$ be the embedding of $R$ in $R_{1}$ and let $\bar{x} \in \nu^{*-1}(x)$. Observe that if $x \in S_{R}-X$ then $x$ does not split in $R_{1}$ since we can construct a model $R_{2}$ of $K_{\overline{C R}}^{*}$ containing $R$ in which $x$ does not split by first constructing $R^{*}$ as in Proposition 2 and then extending $R^{*}$ to a model of $K_{\overline{C R}}$ in which $x$ does not split. Define $\tilde{R}_{1}$ as a ring of functions on $S_{R}$ as follows: for each $a \in R_{1}$ let $\tilde{a}(x)=a\left(\nu^{*-1}(x)\right)$ for $x \in S_{R}-X$ and $\tilde{a}(x)$ $=a(\bar{x})$ for $x \in X ; \widetilde{R}_{1}$ is the ring of functions $\tilde{a}$ for $a \in R_{1}$. It is not hard to see that $R_{1} \simeq \widetilde{R}_{1}^{*}$ (as constructed in Proposition 2). We omit the details. Suppose that $R \subset \bar{R} \vDash K_{\overline{C R}}$. There is an embedding $\phi_{n^{*}} R_{1} \rightarrow \bar{R}^{*}$ since $R_{1}$ is prime over $R$. The correspondence $\widetilde{R}_{1} \ni a \rightarrow a\left(\epsilon \widetilde{R}_{1}^{*}=R_{1}\right) \rightarrow \phi(a)\left(\epsilon \bar{R}^{*}\right)$ $\rightarrow \phi(a) \in \bar{R}$ defines an embedding of $\widetilde{R}_{1}^{1}$ into $\bar{R}$. Hence $\widetilde{R}_{1}$ is the required extension of $R$.

Remark. If $R \models K_{C R}$ has a prime extension to a model of $K_{C R}^{*}$ (or $K_{\overline{C R}}^{*}$ ) then this prime extension is unique. If $S_{R}$ is not perfect then it is not minimal.

Proposition 3 combined with Theoerms 1 and 2 shows that for $R \neq K_{C R}$ to have a prime extension to a model of $K_{\overline{C R}}^{*}$ it is necessary that $R \vDash T$ and that if $\overline{\bar{R}}=\boldsymbol{K}_{0}$ then this condition is also sufficient.

\section{REFERENCES}

1. A. B. Carson, The model completion of the theory of commutative regular rings, J. Algebra 27 (1973), 136-146.

2. J. Lambek, Lectures on rings and modules, Blaisdell, Waltham, Mass., 1966. MR 34 \#5857.

3. S. Lang, Algebra, Addison-Wesley, Reading, Mass., 1965. MR 33 \#5416.

4. L. Lipshitz, Commutative regular rings with integral closure, Notices Amer. Math. Soc. 21 (1974), A-13. Abstract \#74T-E4.

5. L. Lipshitz and D. Saracino, The model companion of the theory of commutative rings without nilpotent elements, Proc. Amer. Math. Soc. 38 (1973), 381-387.

6. A. Macintyre, Model-completeness for sheaves of structures, Fund. Math. 81 (1973), 73-89.

7. G. E. Sacks, Saturated model theory, Benjamin, Reading, Mass., 1972.

8. D. Saracino and V. Weispfenning, Commutative regular rings without prime models, Yale Notes, July 1973.

9. V. Weispfenning, Model-completeness and elimination of quantifiers for subdirect products of structures, J. Algebra (to appear).

DEPARTMENT OF MATHEMATICS, PURDUE UNIVERSITY, WEST LAFAYETTE, INDIANA 47907 